\title{
铑催化芳基硼酸和二氧化碳对丙烯酰胺的区域选择性芳羧化反应
}

\author{
杭炜席婵娟*
}

(清华大学化学系 生命有机磷化学及化学生物学教育部重点实验室 北京 100084)

\section{Rh(I)-Catalyzed Regioselective Arylcarboxylation of Acrylamides with Arylboronic Acids and $\mathrm{CO}_{2}$}

\author{
Hang, Wei Xi, Chanjuan* \\ (Key Laboratory of Bioorganic Phosphorus Chemistry \& Chemical Biology, Ministry of Education, \\ Department of Chemistry, Tsinghua University, Beijing 100084)
}

近些年来, 二氧化碳作为廉价、低毒、丰富和绿色 的一碳合成子，被广泛用来制备高附加值的有机化合 物 $^{[1]}$, 其中, 过渡金属催化或光催化的二氧化碳与烯烃 的氢羧化反应备受关注, 也取得了重要的进展(Scheme $1 \mathrm{a})^{[2-3]}$. 二氧化碳与烯烃的双官能化反应可以合成高官 能化的羧酸衍生物, 为二氧化碳高附加值化带来契机. 尽管过渡金属催化的二氧化碳与烯烃的双官能化反应 已有报道 ${ }^{[4-5]}$, 然而二氧化碳与烯烃的芳羧化反应鲜有 报道. 2019 年, 席婵娟课题组 ${ }^{[6]}$ 报道了卤代烃与二氧化 碳对烯烃的碳羧化反应，首次涉及了烯烃与二氧化碳的 芳羧化反应. 2020 年, 李纲课题组 ${ }^{[7]}$ 报道了光催化的芳 基卤代烃和二氧化碳对苯乙烯衍生物的芳羧化反应 (Scheme 1b), 到目前为止, 这是仅实现二氧化碳对烯烃 芳羧化的一个反应，但是，该类反应的底物依然受限， 无法实现缺电子的 $\alpha, \beta$-不饱和烯烃的芳羧化反应.

中国科学院福建物质结构研究所李纲课题组 ${ }^{[8]}$ 致力 于铑催化的二氧化碳的活化与转化. 近期该课题组通过 使用可能增加铑配合物中间体亲核活性的 $\mathrm{Ag}$ 盐添加剂 (如 $\mathrm{AgOTf} 、 \mathrm{AgCl}$ ), 实现了铑催化的芳基嗍酸和二氧化 碳对丙烯酰胺的芳羧化反应(Scheme 1c). 研究发现通过 加入 $\mathrm{Ag}$ 盐添加剂可以显著抑制单纯芳基化副产物的生 成, 从而得到高比例的双官能团化的目标产物 ${ }^{[9]}$.

在优化的反应条件下, 作者对底物的反应性进行了 拓展, 代表性的底物拓展结果见表 1. 首先, 使用各类 不同的芳基硼酸, 结果发现单取代或多取代的芳基硼酸 都能以不错的收率获得目标产物, 并且吸电子基和给电 子基均可以兼容. 接着, 作者又笁选了各类不同的丙烯 (a) Hydrocarboxylation of alkenes with $\mathrm{CO}_{2}$<smiles>[R]C=C([R])[R]</smiles>

(b) Arylcarboxylation of alkenes with $\mathrm{CO}_{2}$

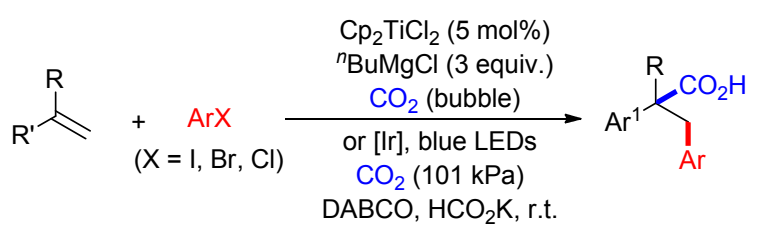

(c) $\mathrm{Rh}(\mathrm{I})$-catalyzed arylcarboxylation of acrylamides with $\mathrm{CO}_{2}$

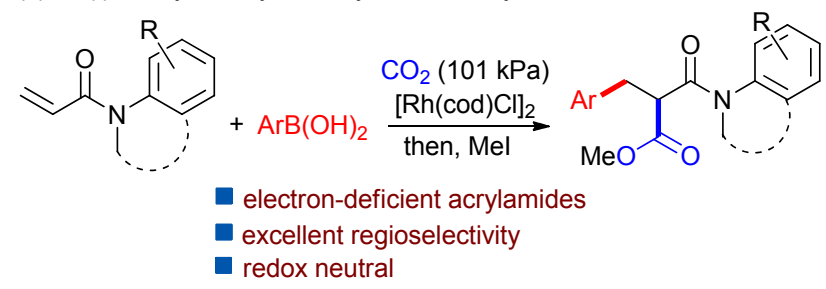

图式 1 二氧化碳与烯烃的羧化反应

Scheme 1 Carboxylation of alkenes with $\mathrm{CO}_{2}$

酰胺衍生物，结果表明使用芳环间位和对位单取代或双 取代的丙烯酰胺都能获得不错的收率，同样吸电子基和 给电子基都可以兼容. 当将丙烯酰胺氮上的甲基换成䒾 基、苯基甚至环化物，仍然可以以较高的收率获得芳羧 化产物。

为了展示该反应的重要性，作者用芳羧化产物进行 进一步衍生化反应研究(Scheme 2), 以 $80 \%$ 的收率制备 了羟吲哚类化合物 $\mathbf{4}$, 该化合物广泛存在于天然产物分

* Corresponding author. E-mail: cjxi@tsinghua.edu.cn. Published online December 30, 2020. 
表 1 反应底物普适性拓展

Table 1 Substrate scope of reaction
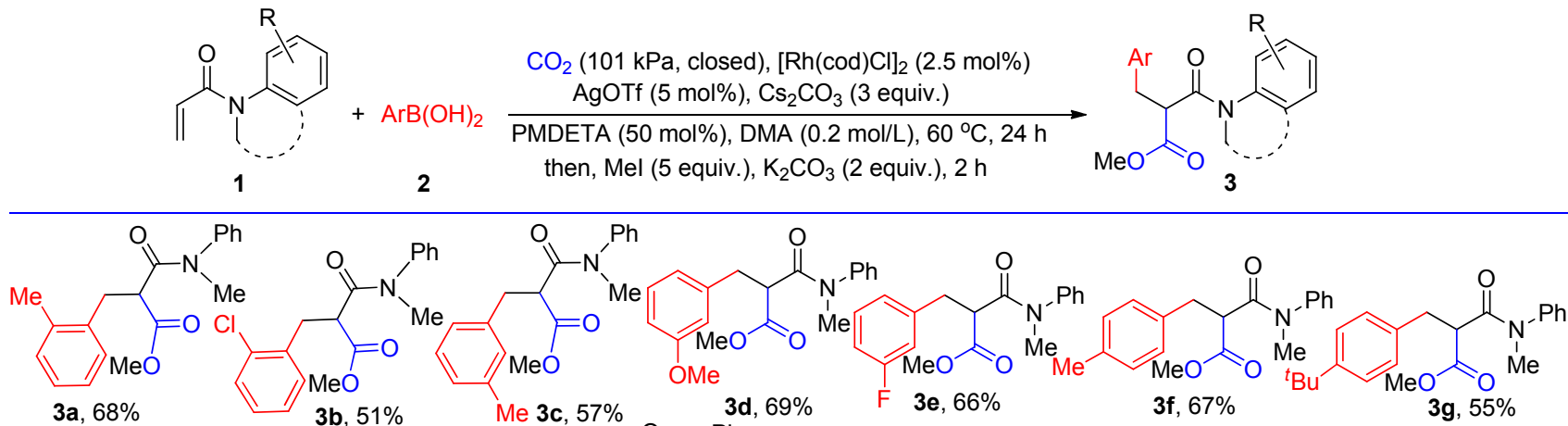
3a, $68 \%$

3b, $51 \%$

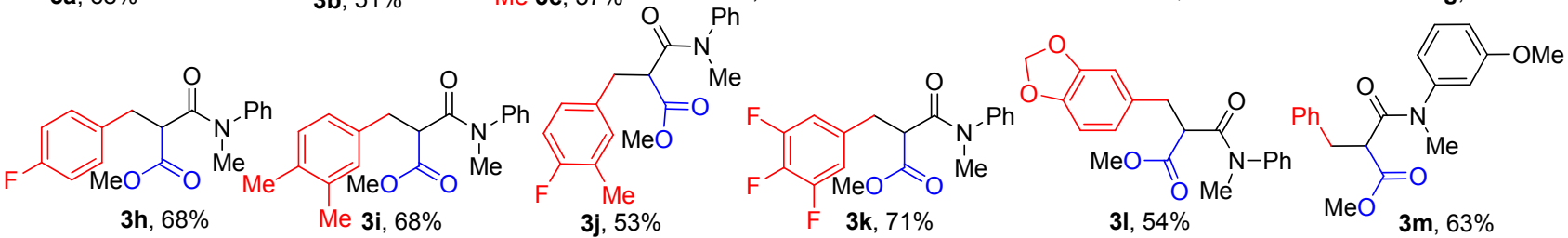
Ph $3 h, 68 \%$ Me $3 \mathbf{i}, 68 \%$

F $3 \mathbf{k}, 71 \%$

$31,54 \%$ $3 m, 63 \%$<smiles>COC(=O)C(Cc1ccccc1)C(=O)N1CCc2ccccc2N1C(=O)C(Cc1ccccc1)C(=O)N1CCCc2ccccc21</smiles>

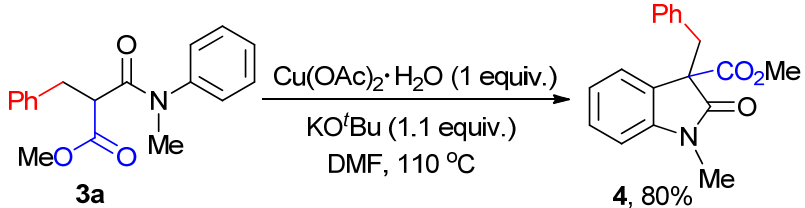

图式 2 芳羧化产物的衍生化反应

Scheme 2 Carboxylation product elaboration
子和药物分子中 ${ }^{[10]}$.

最后作者对该反应的机理进行了深入的探究 (Scheme 3). 首先，作者直接将副产物 5 分别投入标准 反应体系和不加铑催化剂的反应体系中，发现并没有获 得相应的目标产物(Schemes 3a，3b). 这一结果说明，反 应确实连续进行了 $\alpha, \beta$-不饱和烯烃的共轭加成和二氧化 碳的亲核加成，而不是先共轭加成，然后水解，再在碱 (a)<smiles>CN(C(=O)CCc1ccccc1)c1ccccc1</smiles>

(b)<smiles>CN(C(=O)CCc1ccccc1)c1ccccc1</smiles>

(c)<smiles>C=CC(=O)N(C)[Pb]OCCO</smiles>

$\mathrm{CO}_{2}\left(101 \mathrm{kPa}\right.$, closed), $[\mathrm{Rh}(\operatorname{cod}) \mathrm{Cl}]_{2}(2.5 \mathrm{~mol} \%)$ AgOTf (5 mol\%), $\mathrm{Cs}_{2} \mathrm{CO}_{3}$ (1 equiv.)

PMDETA (50 mol\%), DMA (0.2 mol/L), $60^{\circ} \mathrm{C}, 24 \mathrm{~h}$

then, $\mathrm{Mel}$ (5 equiv.), $\mathrm{K}_{2} \mathrm{CO}_{3}$ ( 2 equiv.), $2 \mathrm{~h}$<smiles>CN(C(=O)CCc1ccccc1)c1ccccc1</smiles>

$\mathrm{CO}_{2}(101 \mathrm{kPa}$, closed) $\mathrm{AgCl}(5 \mathrm{~mol} \%), \mathrm{Cs}_{2} \mathrm{CO}_{3}$ (1 equiv.)

PMDETA (50 mol\%), DMA (0.2 mol/L), $60^{\circ} \mathrm{C}, 24 \mathrm{~h}$ then, Mel (5 equiv.), $\mathrm{K}_{2} \mathrm{CO}_{3}$ (2 equiv.), $2 \mathrm{~h}$

$\mathrm{D}_{2} \mathrm{O}$ (3 equiv.), $[\mathrm{Rh}(\mathrm{cod}) \mathrm{Cl}]_{2}(2.5 \mathrm{~mol} \%)$ AgOTf (5 mol\%), $\mathrm{Cs}_{2} \mathrm{CO}_{3}$ (1 equiv.)

PMDETA (50 mol\%), DMA (0.2 mol/L), $60^{\circ} \mathrm{C}, \mathrm{Ar}, 24 \mathrm{~h}$

then, Mel (5 equiv.), $\mathrm{K}_{2} \mathrm{CO}_{3}$ (2 equiv.), $2 \mathrm{~h}$<smiles>CN(C(=O)CCc1ccccc1)c1ccccc1</smiles>

$100 \%$ recovered

图式 3 控制实验

Scheme 3 Control experiments 
的作用下拔氢进攻二氧化碳. 接着, 作者又在氩气氛围 下使用気水淬灭标准反应, 以 $73 \%$ 的收率获得了気代的 化合物 5-D (Scheme 3c). 这一结果表明, 反应体系中确 实存在共轭加成后形成的烯醇铑中间体, 该中间体直接 参与对二氧化碳的亲核加成反应. 这种烯醇铑参与反应 的可能机理对将来研究铑催化的二氧化碳利用具有重 要的启示作用.

综上所述, 李纲课题组首次实现了氧化还原中性条 件下铑催化的芳基嗍酸和二氧化碳对丙烯酰胺的芳羧 化反应, 制备出了一系列可衍生化的高附加值羧酸化合 物. 反应中 $\mathrm{Ag}$ 盐添加剂的加入抑制了容易生成的、经 由铑配合物中间体还原消除产生的单纯芳基化副产物, 显著提高了目标双官能团化产物的产率, 尽管目前 $\mathrm{Ag}$ 盐的具体作用机制还不明了. 该反应表现出了良好的底 物普适性和官能团兼容性, 丰富了二氧化碳与烯烃的双
官能化反应体系, 进一步拓展了二氧化碳的应用.

\section{References}

[1] Wang S.; Xi, C. Chem. Soc. Rev. 2019, 48, 382.

[2] Tortajada, A.; Juliá-Hernández, F.; Börjesson, M.; Moragas, T.; Martin, R. Angew. Chem., Int. Ed. 2018, 57, 15948.

[3] Murata, K.; Numasawa, N.; Shimomaki, K.; Takaya, J.; Iwasawa, N. Chem. Commun. 2017, 53, 3098.

[4] Yan, S.-S.; Fu, Q.; Liao, L.-L.; Sun, G.-Q.; Ye, J.-H.; Gong, L.; Bo-Xue, Y.-Z.; Yu, D.-G. Coord. Chem. Rev. 2018, 374, 439.

[5] Saini S.; Prajapati P. K.; Jain S. L. Cata. Rev. 2020, DOI: 10.1080/01614940.2020.1831757.

[6] Hang, W.; Zou, S.; Xi, C. ChemCatChem 2019, 11, 3814.

[7] Wang, H.; Gao, Y.; Zhou C.; Li, G. J. Am. Chem. Soc. 2020, 142, 8122.

[8] Gao, Y.; Cai, Z.; Li S.; Li, G. Org. Lett. 2019, 21, 3663.

[9] Cai, L.; Fu, L.; Zhou, C.; Gao, Y.; Li, S.; Li, G. Green Chem. 2020, 22,7328 .

[10] Wu, Z. J.; Li, S. R.; Long, H.; Xu, H. C. Chem. Commun. 2018, 54, 4601. 